\title{
Identifying Vegetation from Laser Data in Structured Outdoor Environments
}

\author{
Kai M. Wurm, Henrik Kretzschmar, Rainer Kümmerle, Cyrill Stachniss, Wolfram Burgard \\ University of Freiburg, Dept. of Computer Science, Georges-Köhler-Allee 79, 79110 Freiburg, Germany
}

\begin{abstract}
The ability to reliably detect vegetation is an important requirement for outdoor navigation with mobile robots as it enables the robot to navigate more efficiently and safely. In this paper, we present an approach to detect flat vegetation, such as grass, which cannot be identified using range measurements. This type of vegetation is typically found in structured outdoor environments such as parks or campus sites. Our approach classifies the terrain in the vicinity of the robot based on laser scans and makes use of the fact that plants exhibit specific reflection properties. It uses a support vector machine to learn a classifier for distinguishing vegetation from streets based on laser reflectivity, measured distance, and the incidence angle. In addition, it employs a vibration-based classifier to acquire training data in a self-supervised way and thus reduces manual work. Our approach has been evaluated extensively in real world experiments using several mobile robots. We furthermore evaluated it with different types of sensors and in the context of mapping, autonomous navigation, and exploration experiments. In addition, we compared it to an approach based on linear discriminant analysis. In our real world experiments, our approach yields a classification accuracy close to $100 \%$.
\end{abstract}

Keywords: Vegetation detection, laser reflectivity, self-supervised learning, terrain classification, autonomous navigation

\section{Introduction}

Autonomous outdoor navigation is an active research field in robotics. In most outdoor navigation scenarios such as autonomous wheelchairs, surveillance robots, or transportation vehicles, the classification of terrain plays an important role as most robots have been designed to drive on streets and paved paths rather than on surfaces covered by grass or vegetation. Failing to stay on paved roads introduces the risk of getting stuck and additionally increases wheel slippage, which may lead to errors in the odometry. Therefore, the ability to robustly detect vegetation is important for safe navigation in any of the above-mentioned situations.

In this paper, we propose a novel laser-based terrain classification approach that is especially suited for detecting low vegetation. Such low vegetation typically occurs in structured outdoor environments such as parks or campus sites. Our approach classifies the terrain based on laser scans of the robot's surroundings in order to allow the robot to take the classification result into account during trajectory planning. Low vegetation poses a challenge for laser-based terrain classification since the variance in the measured distances is often small

Preprint submitted to Robotics and Autonomous Systems and thus it is hard to detect it from range measurements alone. Therefore, our approach exploits an effect that is well known from satellite image analysis: Chlorophyll, which is found in living plants, strongly reflects near-IR light [1]. Mobile robots are often equipped with laser range finders such as the popular SICK LMS scanners. These devices emit near-IR light and return the range to the object they measure along with the reflectivity of the surface. Our work formulates the detection of terrain as a classification problem that uses reflectivity, incidence angles, and the measured distances as inputs to identify whether a measured surface corresponds to vegetation. In addition to that, we provide a way to gather training data in a self-supervised way and thus eliminate the cumbersome work of manually labeling training examples.

In this work, we evaluate two sensor setups: rotating laser scanners capturing 3D pointclouds and laser scanners mounted at a fixed angle. We show that classifiers can be trained in a self-supervised way using a vibration-based classification approach to label training data. To integrate classification results into a representation of the environment, we apply a probabilistic mapping method similar to occupancy grid mapping [2].

April 10, 2013 


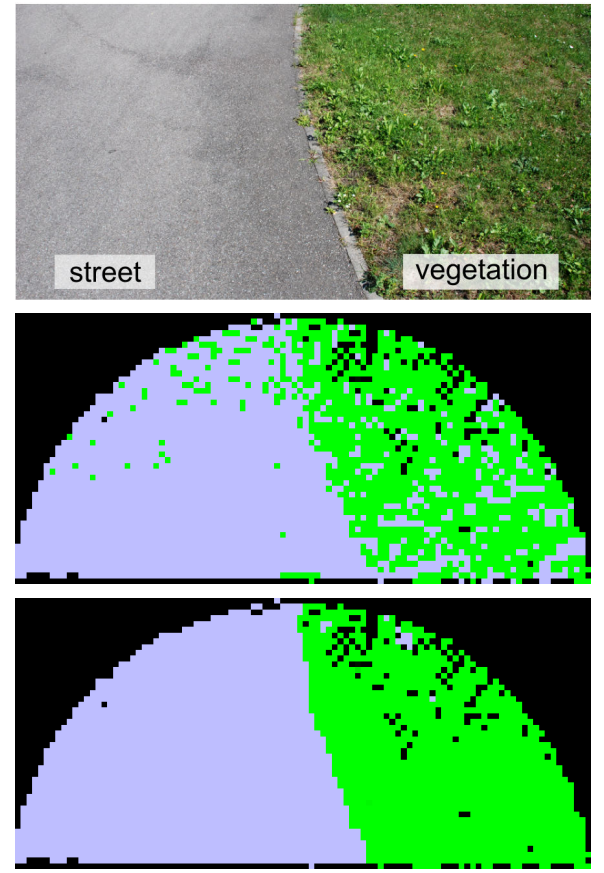

Figure 1: A street and an area containing grass (top) and typical classification results obtained based on range differences (middle) and remission values (bottom). Shown is a bird's eye view of a 3D scan of the area depicted at the top with a maximum range of $5 \mathrm{~m}$. Whereas points classified as street are depicted in blue, points corresponding to vegetation are colored in green.

In our experiments, we illustrate that our approach can be used to accurately map vegetated areas and do so with a higher accuracy than standard techniques that are solely based on range values (see Fig. 1). We furthermore present applications to autonomous navigation in structured outdoor environments in which robots benefit from the knowledge about the vegetation in their surroundings.

This paper is organized as follows. After discussing related work, we will briefly describe support vector machines, which we use for classification. In Sec. 4, we present our approach to terrain classification using laser reflectivity. Sec. 6 describes the mapping approach used to integrate multiple measurements of the same region. Finally, in Sec. 9 we present the experimental results obtained with real data and with real robots navigating through our university campus and neighboring areas.

\section{Related Work}

There exist several approaches for detecting vegetation using laser measurements. Wolf et al. [3] use hidden Markov models to classify scans from a tilted laser scanner into navigable (e.g., street) and non-navigable (e.g., grass) regions. The main feature for classification is the variance in height measurements relative to the robot height. Other approaches analyze the distribution of $3 \mathrm{D}$ endpoints in a sequence of scans $[4,5,6]$. These algorithms are able to detect various types of obstacles such as tree trunks, high grass, or bushes. However, flat vegetation, such as a freshly mowed lawn, cannot be reliably detected using this feature alone.

It seems intuitive to use color cameras to detect vegetation in the environment. Manduchi, for example, uses a combination of color and texture features to detect grass in camera images [7]. The main drawback of using cameras is that they are sensitive to lighting conditions. Shadows, for instance, can have a strong influence on the appearance of vegetation. A more robust classifier can be derived using the Normalized Difference Vegetation Index (NDVI). This value is based on the difference between red and near-infrared light reflectance and is a strong indicator for the presence of vegetation, see [1] for a discussion on this topic. On a mobile robot, the NDVI can be determined using a calibrated combination of a regular and an infrared camera [8] or a single camera that uses a filter mask to capture both frequency bands at different positions on the same sensor [9]. Unfortunately, such a sensor setup is rarely available on a mobile robot and, being a passive sensor, still depends on the presence of ambient light.

A combination of camera and laser measurements has been used to detect vegetation in several approaches [8, $10,11,12]$. In a combined system, Wellington et al. [12] use the remission value of a laser scanner in addition to density features and camera images as a classification feature. However, they do not model the dependency between remission, measured range, and incidence angle. Probably due to this fact, they found the feature to be only "moderately informative". In contrast to that, we show in our work that remission is highly informative if it is considered jointly with the measured range and the incidence angle of the individual laser beams.

The approach that is closest to our approach has been proposed by Bradley et al. [8]. Vegetation is detected using a combination of laser range measurements, regular and near-infrared cameras. Vegetation is recognized using the NDVI computed from both camera images. 3D laser measurements of the environment are projected into the camera images. A classifier is then trained using the vegetation feature and features from the distribution of 3D endpoints. According to the authors, the approach yields a classification accuracy of up to $95 \%$ but requires sophisticated camera equipment. In contrast to such systems that consider cameras in combina- 
tion with laser range finders or infra-red cameras, our approach uses a laser scanner as the sole sensor. Our system is independent of lighting conditions and can be used on a variety of existing robot systems that are often already equipped with a laser range finder. We evaluated our approach in the context of detecting flat vegetation at ranges of up to $5 \mathrm{~m}$. In this setting, we achieved classification results with an accuracy of over $99 \%$ in all our experiments.

Terrain types have also been classified using vibration sensors on a robot $[13,14,15,16]$. In these approaches, the robot traverses the terrain and the induced vibration is measured using accelerometers. The measurements are usually analyzed in the Fourier domain. Sadhukhan et al. [15] presented an approach based on neural networks. A similar approach is presented by DuPont et al. [14]. Brooks and Iagenemma [13] use a combination of principal component analysis and linear discriminant analysis to classify terrain. More recently, SVMs have been used by Weiss et al. [16, 17]. Vibration-based approaches typically offer highly accurate classification results. The drawback of such methods, however, is that only the terrain the robot is moving on can be classified and not the terrain in front of the robot. Thus, this information cannot be integrated well into the path planning system of a mobile robot.

There exist previous approaches that apply selfsupervised learning to classify terrain and detect obstacles. In the context of the LAGR-program (Learning Applied to Ground Robots), a number of methods were developed that exploit terrain knowledge in the surrounding of the robot to predict surface terrain in the far range. These near-to-far approaches use color information [18, 19], 3D geometry information [20], or texture information [21, 22]. Self-supervised learning was also used by Dahlkamp et al. [23] in a vision-based road detection system. Here, laser measurements are used to identify nearby traversable surfaces. This information is then used to label camera image patches in order to train a classifier that is able to predict traversability far away from the robot. In our approach, we adopt the idea of self-supervision to generate labeled training data. We apply a vibration-based classifier to label laser measurements recorded by the robot. This labeled dataset is then used to train a laser-based vegetation classifier. Both classifiers used in our approach have been implemented using support vector machines, which will be introduced in the following section.

This paper extends our previously published conference paper [24]. First, we provide an extended discussion and evaluation including new experiments. Several different sensor types are evaluated and additional large- scale, autonomous navigation and mapping experiments are presented. Second, we provide means for computationally efficient classification so that terrain classification can be carried out on low-powered devices with limited computational resources, which was not directly possible with our previously presented system.

\section{Support Vector Machines}

The support vector machine (SVM) is a kernel-based learning method which is widely used for classification and regression problems [25]. The SVM learns a hyperplane in a higher-dimensional feature space. Two classes of data points are separated by a hyperplane so that the margin between training points and the plane is maximized:

$$
\max _{w \in H, b \in \mathbb{R}} \min _{x_{i} \in \mathcal{D}}\left\{\left\|x-x_{i}\right\| \mid x \in H,\langle w, x\rangle+b=0\right\},
$$

where $H$ is some dot product space, $x_{i} \in \mathcal{D}$ are training points, and $w$ is a weighting vector. The following decision function is used to determine the class label $y$ :

$$
\begin{aligned}
f(x) & =\langle w, x\rangle+b \\
y & =\operatorname{sgn}(f(x))
\end{aligned}
$$

The hyperplane can be constructed by solving a quadratic programming problem. To separate nonlinear classes, the so-called kernel trick is applied. The training data is first mapped into a higher-dimensional feature space using a kernel function. A separating hyperplane is then constructed in this features space which yields a nonlinear decision boundary in the input space. In practice, the Gaussian radial basis function (RBF) is often used as a kernel function. It is given by

$$
k\left(x, x^{\prime}\right)=\exp \left(-\frac{\left(x-x^{\prime}\right)^{2}}{2 l^{2}}\right),
$$

where $l$ is the so-called length-scale parameter.

There exist derivatives of the basic SVM-formulation that are robust against occasional mislabeled training points. Among those, $C$-SVM is a popular method that uses a soft margin. An additional parameter commonly denoted as $C$ adjusts the trade-off between maximizing the margin and minimizing the training error. Throughout this work, we use the $C$-SVM implementation of LibSVM [26].

\subsection{Class Probabilities}

The decision function of support vector machines, as given in Eq. 3, yields a class label $y$ which is not a probability: $y$ is either -1 or 1 . In many cases, however, it 


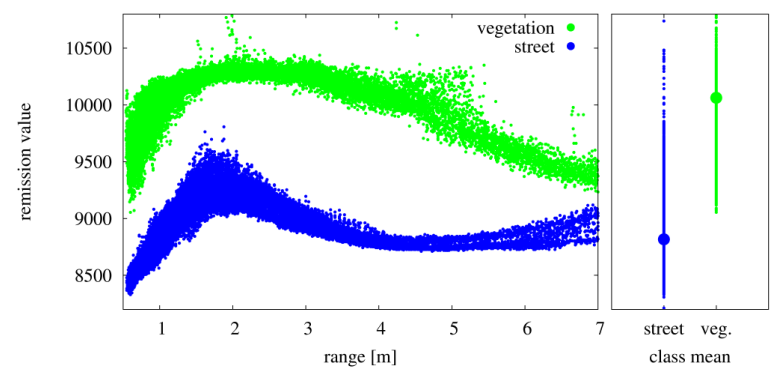

Figure 2: Left: typical remission measurements of a SICK LMS 291S05 for street (blue) and vegetation class (green). Right: mean remission values for both classes.

is desirable to know the uncertainty associated with the output of a classifier. One of these cases is the terrain classification approach discussed in this paper, in which sensor measurements are uncertain and this uncertainty is propagated to the classification results. Knowing the uncertainty associated with classification results enables us to use probabilistic model estimation methods as we will discuss in Sec. 6.

Several methods have been proposed to map the output of SVMs to posterior class probabilities [27, 28]. Among those a popular approach is Platt's method [27]. It fits the posterior using a parametric model. The model is given by

$$
P(y=1 \mid f)=(1+\exp (A f+B))^{-1},
$$

where $f$ is the output of the function given in Eq. 2 . The parameters $A$ and $B$ are determined using maximum likelihood estimation from a training set $\left\{\left(f_{i}, y_{i}\right)\right\}$ consisting of outputs $f_{i}$ and the corresponding class labels $y_{i}$. For details on the optimization step, we refer the reader to the work by Platt [27].

\section{Terrain Classification Using Remission Values}

The goal of our terrain classification algorithm is to precisely identify those parts of the environment that contain vegetation. The classification results have to be obtained early enough so that the robot's navigation system can take it into account, for example, to avoid traversing grass. For this reason, we focus on classifying laser scans of the environment surrounding the robot. We are interested in distinguishing flat vegetation, such as grass, from drivable surfaces such as streets or paved paths. For the sake of brevity, we will call those classes of materials "street" and "vegetation" in the following sections.

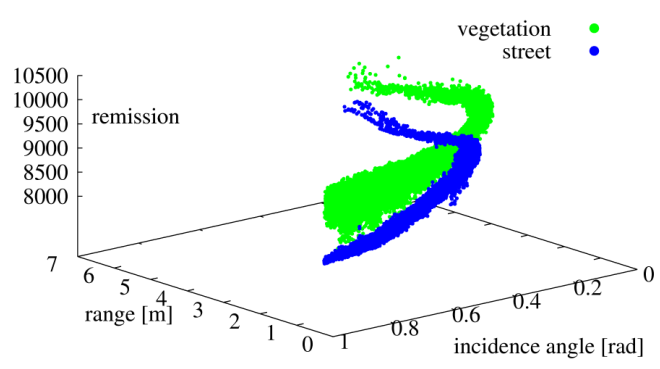

Figure 3: Visualization of sensor data in the full 3D feature space. The plot shows data recorded using a SICK LMS 291-S05.

Typical laser range fingers, such as the SICK LMS scanners or the Hokuyo UTM, emit light at a wavelength of 870 to $915 \mathrm{~nm}$, which is within the nearinfrared range. Chlorophyll, which is found in living plants, strongly reflects near-IR light [1]. We exploit this effect to identify vegetation in measurements of the robots surrounding. Compared to an approach based purely on the scan point distribution, a far better classification accuracy can be achieved when the reflectivity of laser measurements, which is frequently referred to as the remission value is used to classify endpoints. This will be shown in the experiments, see Sec. 9.1.

The remission values returned by the scanner do not only depend on the material of the measured surface, but also on the distance at which it is hit, and on the angle of incidence [29]. This can be seen from Fig. 2, which shows the relation between the measured range and the remission value for both classes. In addition to that, a visualization of the three-dimensional feature space consisting of remission value, distance, and incidence angle is shown in Fig. 3. To obtain highly accurate results, it is important to not only consider the remission values alone. Averaging the remission values over all measured ranges and angles leads to wrong classification results, especially in the near range of up to three meters. This can be seen in Fig. 4. Here, the scene from Fig. 1 was classified using only the mean remission value for both classes ignoring the range and incidence angle.

\subsection{Robust Terrain Classification Using an SVM}

In our approach, we apply a $C$-SVM to classify laser beams depending on the measured range, incidence angle, and remission using a RBF-kernel. The input to the learning algorithm is a labeled set of laser measurements. Each data point consists of a range measure- 


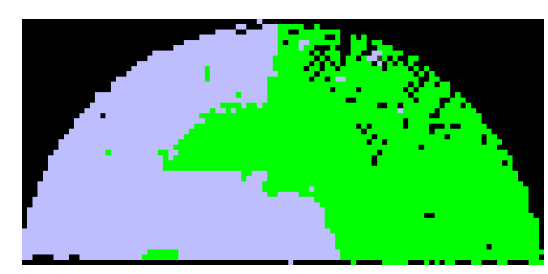

Figure 4: Classification of the scene depicted in Fig. 1 using the mean remission value (see Fig. 2, right) to distinguish both classes.

ment, incidence angle, and a remission value. By determining the separating hyperplane between both data sets, we implicitly model the reflectivity characteristic for the street and vegetation class.

The training is done offline. To optimize the lengthscale parameter $l$ of the kernel used in the SVM as well as the soft-margin parameter $C$, we perform a systematic grid search on the grid $\log _{2} l \in\{-15, \ldots, 3\}$ and $\log _{2} C \in\{-5, \ldots, 15\}$. The optimal parameters are determined using 5-fold cross validation. Once the classifier has been trained, the model can be used on any robot that uses the same sensor in a similar configuration. More specifically, the laser should be mounted so that it measures the surface at angles and distances similar to those observed during the training phase.

\section{Training Data from Vibration-Based Terrain Classification}

In this paper, we propose a self-supervised training method to avoid tedious hand-labeling of dense threedimensional data. We do so by utilizing the output of a second, reliable and pre-trained classifier that uses different sensor modalities to label training data for our laser-based classification problem.

While the robot is moving through the environment, it generates a 3D model using the method described in Sec. 6. To acquire the training set, the remission value, incidence angle, and distance are stored along with each measured scan point. As the robot moves through the environment, it traverses previously scanned surface patches. Those patches are labeled using the outcome of a vibration-based classifier, which is described below, and the labeled data are then fed to the SVM for training. In our current system, training the laser-based classifier is an offline step and we do not consider retraining during runtime.

Since incidence angles cannot be measured directly, they need to be estimated from the measurements. This estimation, however, can be done in a straightforward way. Given a 3D scan, one can group neighboring endpoints and compute the Eigenvalues of the covariance matrix obtained from these points. The direction of the Eigenvector that corresponds to the smallest Eigenvalue is a good estimate of the surface normal. From the surface normal as well as the angle of the laser beam relative to the robot, the incidence angle can be computed.

\subsection{Terrain Classification Based on the Vibration of the Vehicle}

We use a vibration-based classifier to label training data for the laser-based classification system. Different types of terrain cause vibrations of different characteristics. These vibrations can be measured, for example, by an inertial measurement unit (IMU) and can be used to classify the terrain the robot currently traverses. Note that such vibration-based classifiers do not allow the prediction of terrain classes in areas which have not been traversed yet. Thus, it is useful for training, but cannot be used for online classification of the area in front of the robot.

In other works $[13,14,15,16]$, up to seven classes of terrain have been successfully classified. In our system, however, we only need to differentiate between vegetation and streets (non-vegetation) to generate training data for the classifier. In our experiments, we use an XSens MTi to measure the acceleration along the vertical axis. We apply a Fourier transform to the raw acceleration data. In our algorithm, a 128-point FFT is used to capture the frequency spectrum of up to $25 \mathrm{~Hz}$.

The frequency spectrum also depends on the speed of the robot. To account for this dependency, it has been suggested to train several classifiers at different speeds [17]. In our system, however, we decided to treat the forward velocity as a training feature instead. In addition to this, we also use the rotational velocity as a feature to account for vibrations which result from the skid-steering of our robot.

To classify the acceleration and velocity data, we again use a $C$-SVM with a RBF-kernel. Our feature vector $x$ consists of the first 32 Fourier magnitudes $\left|F_{m}\right|$, the mean forward velocity $\bar{v}_{t}$, and the mean rotational velocity $\bar{v}_{r}$ of the robot over the sample period

$$
\begin{aligned}
\left|F_{m}\right| & =\sqrt{\left(\operatorname{Re} F_{m}\right)^{2}+\left(\operatorname{Im} F_{m}\right)^{2}}, m \in\{0, \ldots, 31\} \\
x & =\left\{\left|F_{0}\right|, \ldots,\left|F_{31}\right|, \bar{v}_{t}, \bar{r}_{t}\right\},
\end{aligned}
$$

where $F_{m}$ denotes the $m$-th Fourier component. The classifier is trained by recording short tracks of about $50 \mathrm{~m}$ at varying speeds both on streets and on vegetation. Parameter optimization was done using grid search and 5-fold cross validation. In our experiments, we achieved an almost perfect classification accuracy with this vibration-based approach and thus this data is well 


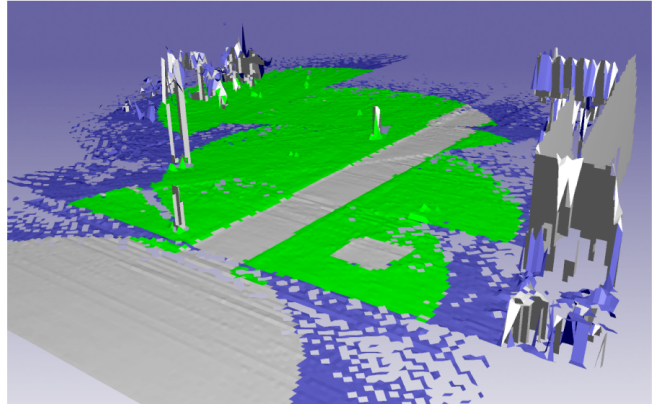

Figure 5: Visualization of a 3D model with vegetation highlighted in green.

suited to label the training data for the laser-based classification.

\section{Mapping Vegetation}

We use multi-level surface maps [30] to model the environment. This representation uses a $2 \mathrm{D}$ grid and in each cell stores a set of patches representing individual surfaces at different heights. In our approach, we maintain the probability of vegetation for each surface patch. Let $P\left(\mathrm{v}^{i}\right)$ denote this probability for patch $i$. In general, surface patches will be observed multiple times. For this reason, we need to probabilistically combine results from several measurements. In this way, the uncertainty in classification is explicitly taken into account.

Let $z_{t}$ be a laser measurement at time $t$. Analogous to Moravec [2], we obtain an update rule for $P\left(\mathrm{v}^{i} \mid z_{1: t}\right)$. First, we apply Bayes' rule and obtain

$$
P\left(\mathrm{v}^{i} \mid z_{1: t}\right)=\frac{P\left(z_{t} \mid \mathrm{v}^{i}, z_{1: t-1}\right) P\left(\mathrm{v}^{i} \mid z_{1: t-1}\right)}{P\left(z_{t} \mid z_{1: t-1}\right)} .
$$

We then compute the ratio

$$
\frac{P\left(\mathrm{v}^{i} \mid z_{1: t}\right)}{P\left(\neg \mathrm{v}^{i} \mid z_{1: t}\right)}=\frac{P\left(z_{t} \mid \mathrm{v}^{i}, z_{1: t-1}\right)}{P\left(z_{t} \mid \neg \mathrm{v}^{i}, z_{1: t-1}\right)} \frac{P\left(\mathrm{v}^{i} \mid z_{1: t-1}\right)}{P\left(\neg \mathrm{v}^{i} \mid z_{1: t-1}\right)} .
$$

Similarly, we obtain

$$
\frac{P\left(\mathrm{v}^{i} \mid z_{t}\right)}{P\left(\neg \mathrm{v}^{i} \mid z_{t}\right)}=\frac{P\left(z_{t} \mid \mathrm{v}^{i}\right)}{P\left(z_{t} \mid \neg \mathrm{v}^{i}\right)} \frac{P\left(\mathrm{v}^{i}\right)}{P\left(\neg \mathrm{v}^{i}\right)},
$$

which can be transformed to

$$
\frac{P\left(z_{t} \mid \mathrm{v}^{i}\right)}{P\left(z_{t} \mid \neg \mathrm{v}^{i}\right)}=\frac{P\left(\mathrm{v}^{i} \mid z_{t}\right)}{P\left(\neg \mathrm{v}^{i} \mid z_{t}\right)} \frac{P\left(\neg \mathrm{v}^{i}\right)}{P\left(\mathrm{v}^{i}\right)} .
$$

Applying the Markov assumption that the current observation is independent of previous observations given we know that a patch contains vegetation gives

$$
P\left(z_{t} \mid \mathrm{v}^{i}, z_{1: t-1}\right)=P\left(z_{t} \mid \mathrm{v}^{i}\right),
$$
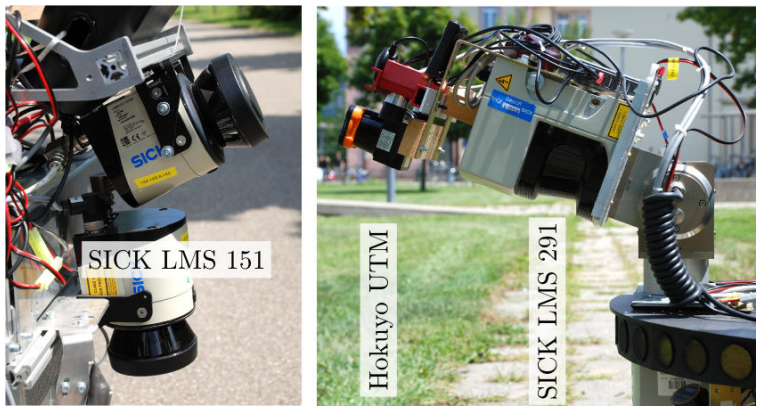

Figure 6: Sensors used in the evaluation.

and utilizing the fact that $P\left(\neg \mathrm{v}^{i}\right)=1-P\left(\mathrm{v}^{i}\right)$, we obtain

$$
\begin{aligned}
& \frac{P\left(\mathrm{v}^{i} \mid z_{1: t}\right)}{1-P\left(\mathrm{v}^{i} \mid z_{1: t}\right)}= \\
& \quad \frac{P\left(\mathrm{v}^{i} \mid z_{t}\right)}{1-P\left(\mathrm{v}^{i} \mid z_{t}\right)} \frac{P\left(\mathrm{v}^{i} \mid z_{1: t-1}\right)}{1-P\left(\mathrm{v}^{i} \mid z_{1: t-1}\right)} \frac{1-P\left(\mathrm{v}^{i}\right)}{P\left(\mathrm{v}^{i}\right)} .
\end{aligned}
$$

This equation can be transformed into the following update formula:

$$
\begin{gathered}
P\left(\mathrm{v}^{i} \mid z_{1: t}\right)= \\
{\left[1+\frac{1-P\left(\mathrm{v}^{i} \mid z_{t}\right)}{P\left(\mathrm{v}^{i} \mid z_{t}\right)} \frac{1-P\left(\mathrm{v}^{i} \mid z_{1: t-1}\right)}{P\left(\mathrm{v}^{i} \mid z_{1: t-1}\right)} \frac{P\left(\mathrm{v}^{i}\right)}{1-P\left(\mathrm{v}^{i}\right)}\right]^{-1}}
\end{gathered}
$$

To perform the update step, we need an inverse sensor model $P\left(\mathrm{v}^{i} \mid z\right)$. This, however, can directly be obtained from the estimated class probabilities of the classifier. During the training phase, we apply Platt's method as discussed in Sec. 3.1. The posterior class probabilities computed using Eq. 5 constitute an inverse sensor model and in this way the uncertainty in classification is explicitly taken into account.

The prior probability of a patch to contain vegetation $P\left(\mathrm{v}^{i}\right)$ clearly depends on the environment that the robot is operating in. In our system, it was set to 0.5 to not preference any class. This seems reasonable in an environment such as the one depicted in Fig. 12 but can be adapted if this is needed.

\section{Comparison of Different Sensors}

Most laser range finders that are used on mobile robots are able to provide range and remission information and all of these sensors measure surfaces using near-infrared light. Thus our approach can be applied to data from any such sensor as long as it provides remission values. We evaluated three different laser scanners under comparable conditions. The sensors we evaluated were the SICK LMS 291-S05, the SICK LMS 151, and 

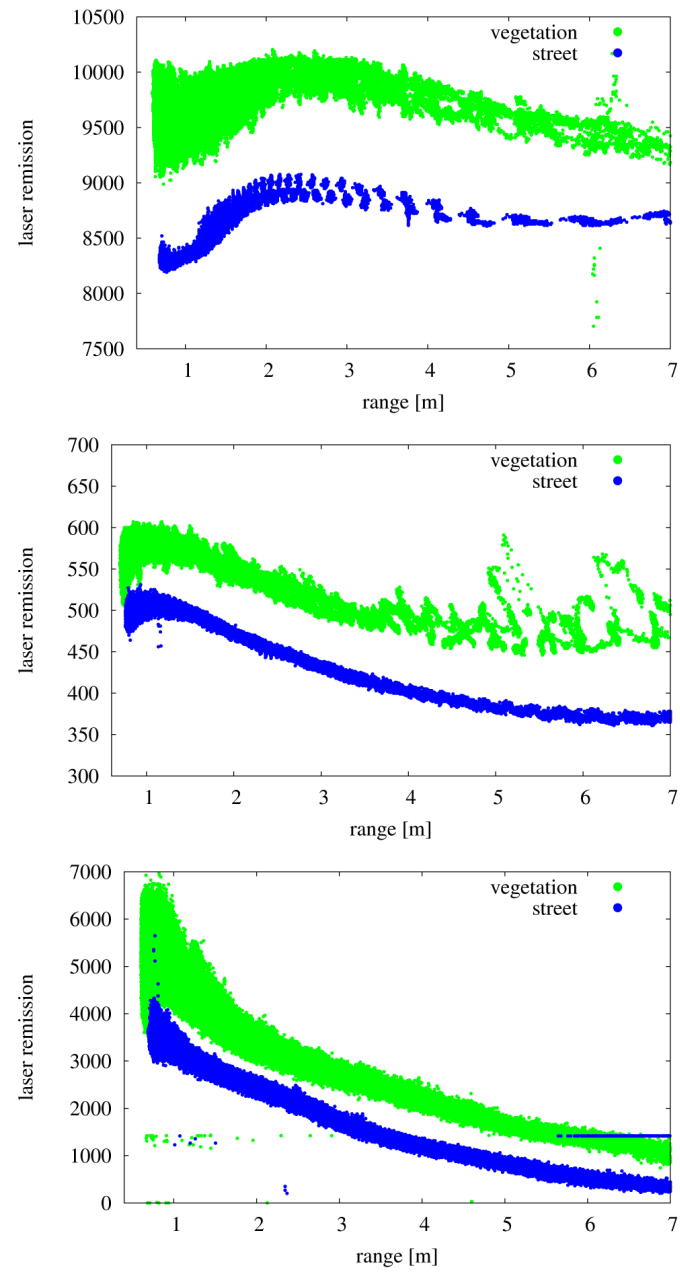

Figure 7: Comparison of remission values of a SICK LMS 291 (top), SICK LMS 151 (middle), and a Hokuyo UTM 30LX (bottom). Please note that these are projections of the full three-dimensional data sets since incidence angles are not shown.

the Hokuyo UTM 30LX (see Fig. 6). Each scanner was mounted on a mobile robot at a fixed angle of 70 degrees relative to the ground and was mounted at a height of approximately $0.6 \mathrm{~m}$. We recorded range and remission data by moving the robot so that the sensors were swept over a street and areas that contained vegetation. ${ }^{1}$

Example data from the sensors of both classes (projected into the two dimensional space for a better visualization) is depicted in the plots in Fig. 7. It can be seen that all sensors report a considerably higher reflectivity for vegetation than for the street. In this projection, the LMS 291 offers the best separation of the classes, es-

\footnotetext{
${ }^{1}$ Data available at:

http://ais.informatik.uni-freiburg.de/remission-data
}

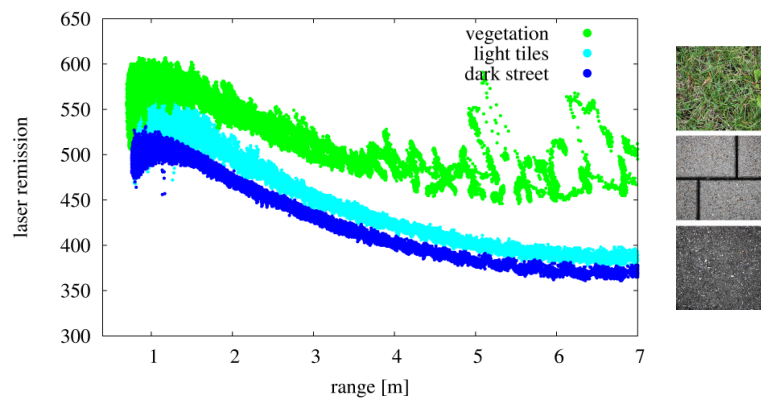

Figure 8: Left: remission values of a SICK LMS 151 measuring vegetation, light tiles, and an asphalt street. Right: examples of the measured surfaces.

pecially in the near range, followed by the LMS 151 . Using the Hokuyo UTM, measurements of both classes are not well separated in the near range of up to approximately one meter.

It is worth mentioning that the measured difference in the reflectivity is not due to the lighter color of grass compared to a dark street. In Figure 8, remission data from a surface covered with light tiles is shown. These tiles exhibit a visual brightness similar to grass. It can be seen that the measured infrared reflectivities are higher than those of an asphalt street but still considerably lower than those of vegetation.

From the examples shown above, one can see that all three sensors can be used for vegetation detection, but the classification accuracy may vary. Depending on the type of sensor, a minimum distance to the ground should be kept for reliable results since in this area, it may be challenging to separate the classes.

\section{Classification for Resource Constrained Systems}

On systems with limited computational resources, such as embedded systems, the SVM classifier can be computationally too demanding to be used online. The computational complexity of the SVM results from the evaluation of the kernel and the potentially high number of support vectors that are needed to appropriately separate the classes. For each laser scan, assuming a frequency of $36 \mathrm{~Hz}$ and 180 laser beams per scan, 6,480 classifications per second need to be performed.

To reduce the computational complexity, we apply linear discriminant analysis [31] to project the threedimensional feature space (range, incidence angle, remission) down to one dimension such that the two classes are best separated. Given this projection, we can then apply an efficient linear classifier to separate the classes and to compute class probabilities for laser 


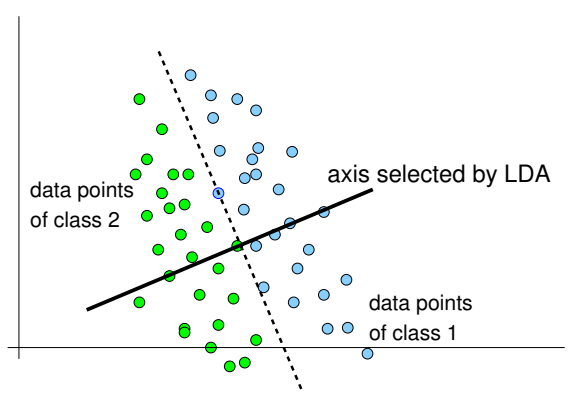

Figure 9: Reduction from $\mathbb{R}^{2}$ to $\mathbb{R}$ using LDA. LDA seeks to separate the two classes (illustrated by green and blue) as well as possible.

measurements based on a Gaussian assumption. The individual steps are explained in the remainder of this section.

\subsection{Linear Discriminant Analysis}

Linear discriminant analysis (LDA) is a supervised dimensionality reduction technique that is similar to PCA. In contrast to PCA, LDA allows to exploit the labels of the datapoints and seeks to estimate a reduced space so that the classes are best separated. Reducing the space to a one-dimensional one, then allows to separate the classes with a naive Bayesian classifier. An illustration of a reduction from $\mathbb{R}^{2}$ to $\mathbb{R}$ is shown in Figure 9. We refer to Alpaydin [31] for more details.

LDA works as follows. Let $K$ be the number of classes $C_{k}$ and $x_{i}$ the $d$-dimensional inputs, here $K=2$ and $d=3$. Let furthermore $t$ be the dimensionality of the target space, in our experiments we found a onedimensional target space to be sufficient. The objective is to find a $d \times t$ matrix $W$ so that $v_{i}=W^{T} x_{i}$ with $v_{i} \in \mathbb{R}^{t}$ and so that the classes $C_{k}$ are separated best in terms of distances between the $v_{i}$.

Let $r_{k, i}$ be an indicator variable with $r_{k, i}=1$ if $x_{i} \in C_{k}$ and 0 otherwise. Let $m_{k}$ be the mean of $d$-dimensional vectors $x_{i} \in C_{k}$. Then, the so-called total within-scatter matrix is given by

$$
S_{W}=\sum_{k=1}^{K}\left(\sum_{i} r_{k, i}\left(x_{i}-m_{k}\right)\left(x_{i}-m_{k}\right)^{T}\right),
$$

and the between-class scatter matrix by

$$
S_{B}=\sum_{k=1}^{K}\left(\sum_{i} r_{k, i}\right)\left(m_{k}-m\right)\left(m_{k}-m\right)^{T},
$$

with

$$
m=\frac{1}{K} \sum_{k=1}^{K} m_{k}
$$

Now let us consider the scatter matrices after projection using $W$. The between-class scatter matrix after projection is $W^{T} S_{B} W$, and the within-scatter matrix accordingly, both are $t \times t$ dimensional. The goal is to determine $W$ in a way that the means of the classes $W^{T} m_{k}$ are as far apart from each other as possible while the spread of their individual projected class samples is small. Similar to covariance matrices, the determinant of a scatter matrix characterizes the spread and it is computed as the product of the eigenvalues specifying the variance along the eigenvectors. Thus we aim at finding the matrix $W$ that maximizes

$$
J(W)=\frac{\left|W^{T} S_{B} W\right|}{\left|W^{T} S_{W} W\right|} .
$$

The largest eigenvectors of $S_{W}^{-1} S_{B}$ are the solution to this problem.

\subsection{Classification Using Linear Discriminant Analysis}

Given the projection computed by LDA, we use an efficient linear classifier to compute class probabilities for laser measurements. For each class $C_{k}$, our method fits a Gaussian distribution, $p\left(x \mid C_{k}\right)$, to the onedimensional data using maximum likelihood estimation. Using Bayes' theorem, we obtain the posterior probabilities

$$
p\left(C_{k} \mid x\right)=\frac{p\left(x \mid C_{k}\right) p\left(C_{k}\right)}{\sum_{i} p\left(x \mid C_{i}\right) p\left(C_{i}\right)},
$$

where $p\left(C_{i}\right)$ is the class prior for class $C_{i}$. Since in our case, we have two classes and a uniform prior, this turns into

$$
p\left(C_{k} \mid x\right)=\frac{p\left(x \mid C_{k}\right)}{p\left(x \mid C_{1}\right)+p\left(x \mid C_{2}\right)},
$$

with $k \in\{1,2\}$, referring to the classes vegetation and streets. Note that this simplified classification approach is significantly faster than the SVM, but it is also less powerful. Our evaluation in Sec. 9.6 shows that the resulting error depends on the type of sensor and the measurement range. In our experiments, we observed that the precision dropped from $99 \%$ down to $96 \%$ in the worst case compared to the SVM-based classifier while LDA-based system was around two orders of magnitude faster than the SVM in our settings.

\section{Evaluation}

Our approach has been implemented and evaluated in several experiments. The experiments are designed to demonstrate that our approach improves navigation 


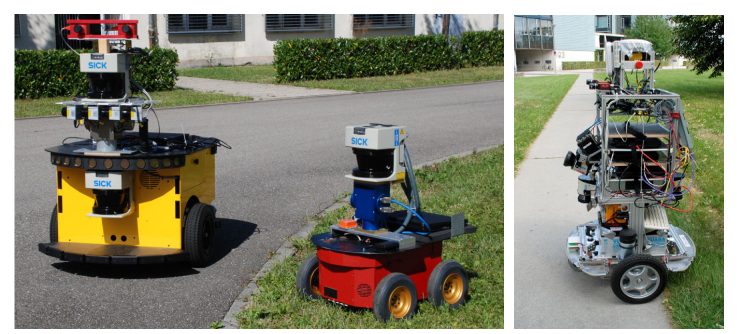

Figure 10: Robots used in our experiments. Left: An ActiveMedia Powerbot (yellow) and a Pioneer2 AT (red). Right: the EUROPA platform.

in structured outdoor environments by enabling robots to reliably detect vegetation.

We used three different robot systems (see Fig. 10). The self-supervised learning approach is evaluated using an ActivMedia Pioneer2 AT, which is able to traverse low vegetation. For mapping large environments and for an autonomous driving experiment, we use an ActivMedia Powerbot platform. This robot cannot safely traverse grass since its castor wheels will block the robot due to its weight. Both robots are equipped with SICK LMS S291-S05 laser scanners on pan-tilt units. In addition to that, the Pioneer robot carries an XSens MTi IMU to measure vibrations. Threedimensional scans are gathered by tilting the laser scanner from 50 degrees upwards to 30 degrees downwards. To evaluate classification on a SICK LMS 151 sensor mounted at a fixed angle, we use the custom-made EUROPA robot platform depicted in Fig. 10 (right). Just as the Powerbot, this platform cannot safely traverse vegetation.

We limit the classification to scans with a range smaller than 5.0 meters. The approach itself is not limited in range. However, due to the coarse angular resolution of the laser scanners and a low mounting height of the sensor relative to the ground (approximately 0.5$0.6 \mathrm{~m}$ ), long range data is too sparse both to gather training data and to reliably detect drivable surfaces and obstacles.

In our current implementation, structures that can be reliably detected as obstacles are not considered in the classification step. This decision is made based on the height difference between potential obstacles and their surrounding. In our experiments, we set this threshold to $0.1 \mathrm{~m}$. Such obstacles are not traversable regardless of whether they are covered by vegetation or not. Note that the classification approach itself would even work on arbitrary surfaces given the approximate knowledge of the surface normal.

\subsection{Comparison to Vegetation Detection Using Range Differences}

In a first experiment, we implemented a vegetation detection algorithm based purely on the range differences of neighboring laser measurements similar to the one proposed by Wolf et al. [3]. This method is only used as a comparison with our proposed classification approach. Three-dimensional data are acquired by gathering a sweep of 2D scans. For each 2D scan, the 2D endpoints $p_{i}=\left\langle x_{i}, y_{i}\right\rangle$ are computed from the range and angle measurements $\left\langle r_{i}, \alpha_{i}\right\rangle$. A local feature $d_{i}$ is then determined for every scan point

$$
d_{i}=x_{i}-x_{i-1} .
$$

This feature captures the local roughness of the terrain. To cope with flat but tilted surfaces, we classify scans based on the absolute difference in $d_{i}$ between adjacent range measurements, as suggested in [11]. Furthermore, we also use the measured range as a training feature to account for the varying data density from near to far scans. We use an SVM to train a classifier based on these features.

In our experiments, we achieve a classification accuracy of about $75 \%$ using the described method. An example of the classification results can be seen in Fig. 1. Similar results have been reported by other researchers [8]. While this result certainly shows that geometric features are useful to detect vegetation, we will show that a substantially higher accuracy can be achieved in case of detecting flat vegetation in the surrounding of the robot.

\subsection{Self-Supervised Learning}

To train our remission-based classifier, we joysticked the Pioneer AT robot through an outdoor environment consisting of a street and an area covered with grass. We acquired 3D scans approximately every $4 \mathrm{~m}$. While the robot was driving, the IMU measured the vibration of the robot. To correct odometry errors of the robot, we employed a 2D SLAM approach [32] and its freely available implementation [33] in combination with 3D scan-matching. We trained our classifier using the selfsupervised approach described in Sec. 4.1. The training set is visualized in Fig. 11a. The data recorded at the border region between street and vegetation were ignored since the precise location of the border cannot be determined using the vibration sensor. The model for the laser-based classifier was trained using 19,989 vegetation and 11,248 street samples.

To evaluate the precision of the classifier, we recorded separate test data at a different location (see 

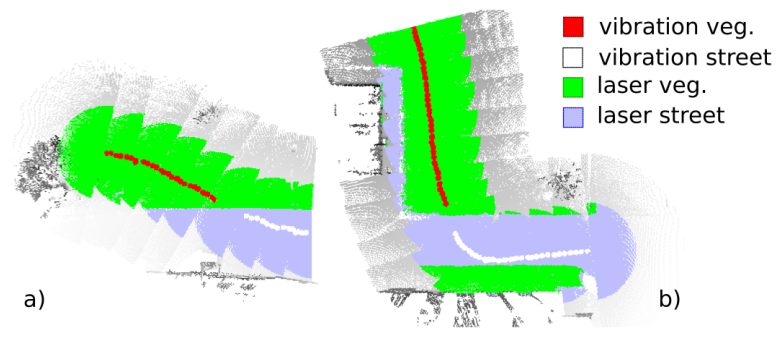

Figure 11: Self-supervised learning. Left: the data set which is used to train the classifier. Right: test set recorded at a different location.

Table 1: Confusion matrix for Exp. 9.2 (number of data points)

\begin{tabular}{|l|r|r|}
\hline & vegetation & street \\
\hline vegetation & 36,300 & 4 \\
\hline street & 138 & 28,745 \\
\hline
\end{tabular}

Fig. 11b and Fig. 12). The test set contains 36,304 vegetation and 28,883 street measurements. Again, the labeling of the data was carried out using the vibration-based classifier. The previously trained classifier reached a recall of $99.6 \%$ and a precision of $99.9 \%$ on the test data. The confusion matrix is given in Table 1. Note that such accurate classification results are not due to overfitting and very similar results are also obtained on other test sets.

\subsection{D Mapping}

In this experiment, we steered the Powerbot robot across the computer science campus at the University of Freiburg. The scanning laser of the robot was tilted to a fixed angle of 20 degrees downwards. In this way, a fairly large area could be mapped in less than 15 minutes. The length of the trajectory is $490 \mathrm{~m}$. The vegetation classifier was used to map vegetation in the threedimensional model of the environment. To properly integrate multiple measurements, we used the mapping approach described in Sec. 6 with a cell size of $0.1 \mathrm{~m}$.

Due to a significantly different hardware setup as compared to the Pioneer AT, we were not able to use the model generated in Sec. 9.2. Instead, we recorded a training set of 12,153 grass and 10,448 street samples by placing the robot in front of flat areas containing only street and only vegetation. This method is only applicable if such training data can be gathered and thus should be considered inferior to the self-supervised approach described in Sec. 4.1.

Compared to the aerial image of the campus site in Fig. 12, the mapping result shown in Fig. 13 is highly accurate. Even small amounts of vegetation, for example between tiles on a path, can be identified. To evaluate the accuracy of the map created during this exper-

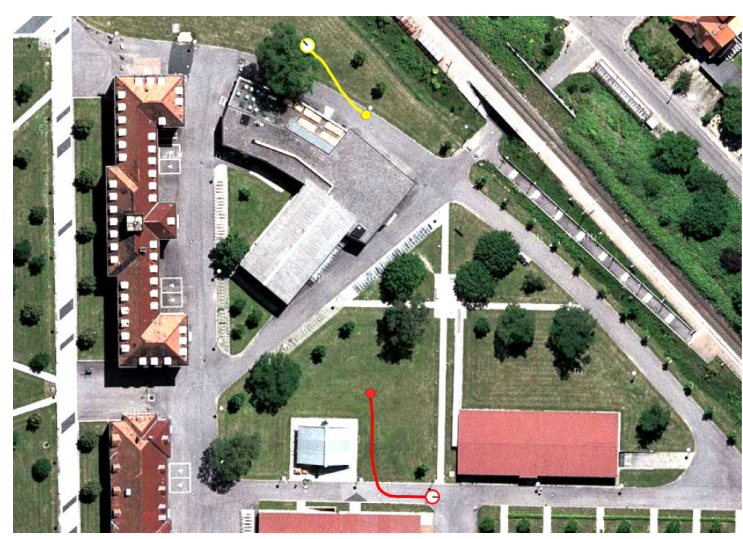

Figure 12: Aerial view of the computer science campus in Freiburg. Estimated robot trajectories are shown for the training (top, yellow) and the test set (bottom, red). Courtesy of Google Maps, Copyright 2008, DigitalGlobe.

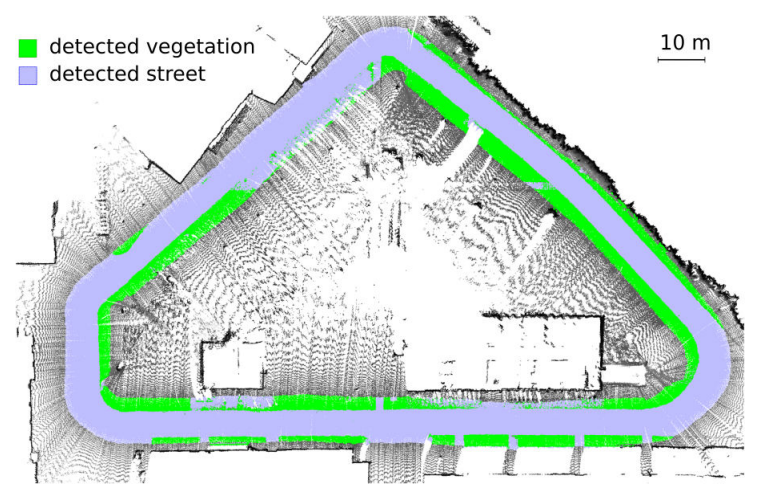

Figure 13: Mapping a large outdoor environment. The laser was tilted at a fixed angle of 20 degrees while the robot was moving. The figure shows a $2 \mathrm{D}$ projection of the $3 \mathrm{D}$ map.

iment, we manually marked wrongly classified cells in a $2 \mathrm{D}$ projection of the $3 \mathrm{D}$ map using our knowledge of this environment. Of a total of 271,638 cells $(75,622$ vegetation, 196,016 street), we found 547 false positives and 194 false negatives. This corresponds to a precision of $99.23 \%$ and a recall of $99.74 \%$.

\subsection{Autonomous Navigation Using a 3D Scanner}

As mentioned above, the Powerbot cannot safely traverse vegetated areas. In the experiment depicted in Fig. 14, the robot was told to navigate to a goal position $80 \mathrm{~m}$ in front of it while avoiding vegetation. Since the robot did not have a map, it explored the environment in the process of reducing its distance to the goal location. Thereby, it used the vegetation classifier to detect vegetation. The environment was represented as described in Sec. 6. Without knowledge about the specific terrain, the shortest obstacle-free path would have led the 


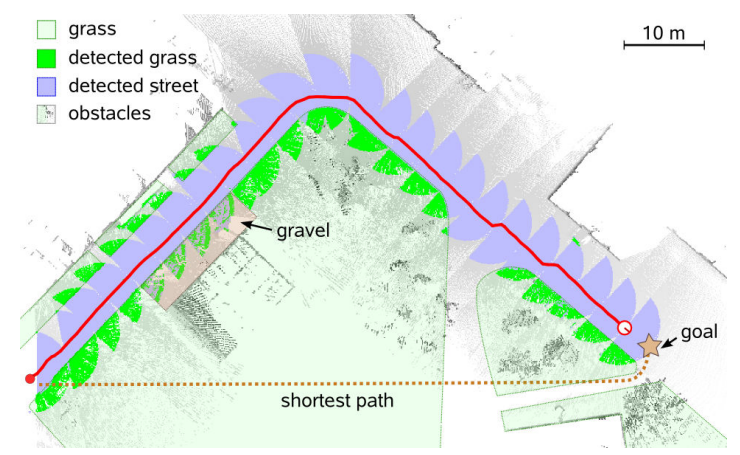

Figure 14: Autonomous navigation experiment. Although the shortest obstacle-free path from the start to the goal position led over grass, the robot reliably avoided the vegetated areas by using our vegetation classifier and traveled over the paved streets to reach its goal.

robot across a large area containing grass. By considering the classification results in the path costs, however, the planner chose a safe trajectory over the street.

\subsection{Autonomous Navigation Using a Fixed-Angle Sen- sor}

To obtain a map of the campus surroundings, the custom-made EUROPA platform depicted in Fig. 10 (right), equipped with a SICK LMS 151 mounted at a fixed angle, was steered along a 7,500 $\mathrm{m}$ trajectory through the campus and parts of Freiburg. Additionally, the data collected by a horizontally mounted range finder are considered for building a 2D occupancy grid. The mapped area combines the computer science campus, urban areas, and the park area of a Freiburg University hospital.

We trained the classifier using data recorded by placing the robot in front of grassland and on a street. Using the collected data, our mapping approach presented in Sec. 6 was able to obtain a map in which the drivable street and non-traversable vegetation were accurately labeled. Fig. 15 visualizes the resulting map which was used to carry out an autonomous navigation experiment. At the beginning of the experiment, the robot was located on the campus in the top left of the map. The goal location was within the park area in the lower right part of the map. By taking into account the classification result of our approach, the robot was able to plan a feasible and safe path towards the goal location. While navigating along the planned path the robot used its horizontally mounted range finder to localize itself given the occupancy map. The robot autonomously traveled a total distance of approximately $1,120 \mathrm{~m}$ and successfully reached the goal location. The trajectory that the robot followed is shown as a red line in Fig. 15. Without

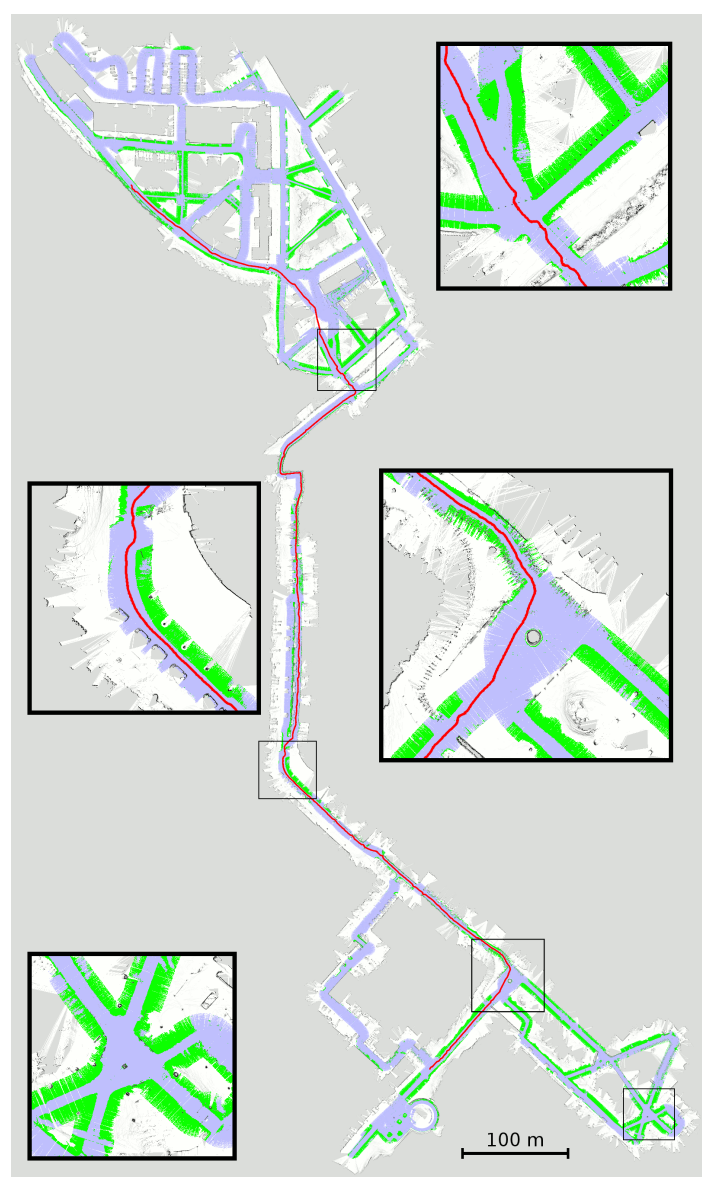

Figure 15: Autonomous navigation experiment. The robot planned a path on a map annotated by our approach. The robot autonomously traveled a distance of approximately $1,120 \mathrm{~m}$. Each black rectangle shows a magnified view of a particular area of the environment.

classifying the vegetation the shortest path would have led partially across large areas covered with vegetation which induces a high risk of getting stuck.

\subsection{Resource-Friendly Classification with Linear Dis- criminant Analysis}

In this section, we compare the accuracy of the SVM and linear discriminant analysis. The LDA approach is less computationally demanding and thus is useful for resource-constraint systems or for robots that run a fairly large number of processes. We evaluated both approaches on data that we recorded with three different laser scanners, i.e., SICK LMS 151, SICK LMS 291, and Hokuyo UTM 30LX.

We divided each dataset into a training dataset containing 2,000 randomly sampled data points and a test dataset containing all the remaining data points. The results are depicted in Tab. 2. Using the LDA approach 
Table 2: Comparison between the SVM-based and the LDA-based classifiers

\begin{tabular}{|l||r|r||r|r|}
\hline & $\begin{array}{r}\text { precision } \\
\text { (SVM) }\end{array}$ & $\begin{array}{r}\text { recall } \\
\text { (SVM) }\end{array}$ & $\begin{array}{r}\text { precision } \\
\text { (LDA) }\end{array}$ & $\begin{array}{r}\text { recall } \\
\text { (LDA) }\end{array}$ \\
\hline LMS 151 & $99.7 \%$ & $100 \%$ & $99.1 \%$ & $99.8 \%$ \\
\hline LMS 291 & $99.9 \%$ & $100 \%$ & $99.4 \%$ & $100 \%$ \\
\hline UTM & $99.9 \%$ & $99.9 \%$ & $96.3 \%$ & $99.8 \%$ \\
\hline
\end{tabular}

on the SICK LMS 151 dataset resulted in a recall of $99.1 \%$ and a precision of $99.8 \%$, whereas the SVMbased classifier gave a recall of $99.7 \%$ and a precision of $100.0 \%$. Using the SICK LMS 291 dataset, the LDAbased approach yielded a recall of $99.4 \%$ and a precision of $100.0 \%$, whereas the use of the SVM resulted in a recall of $99.9 \%$ and in a precision of $100.0 \%$. When applied to the Hokuyo UTM 30LX dataset, the LDAbased approach reached a recall of $96.3 \%$ and a precision of $99.8 \%$, while the SVM-based classifier gave a recall of $99.9 \%$ and a precision of $99.9 \%$.

These results show that both approaches achieve a highly accurate classification. In all cases, the SVMbased approach outperforms the LDA-based method. The relative difference, however, is small. When analyzing the errors, we observed that most of the misclassification of the LDA-based classifier occur at range measurements below $2 \mathrm{~m}$. This means that the system potentially makes poor predictions when classifying areas close to the robot, which may cause the navigation system to stop the vehicle to avoid potential obstacles. Therefore, the highly accurate results of the SVM-based system are preferable for online navigation tasks.

We also compared the runtimes of the SVM and the LDA-based approach. In our experiments, the LDAbased approach was around 400 times faster than the classification using the support vector machine on a regular desktop computer. The SVM used 21 support vectors to separate the training data in this evaluation.

\section{Conclusion}

In this paper, we presented a novel approach to vegetation detection that uses the remission values of laser scanners. Laser remission values depend on the surface reflectivity as well as on the distance and the angle of incidence. This dependency has not been considered by previous methods that detect vegetation. Our approach trains a classifier based on individual laser measurements consisting of the remission value, the distance to the surface, and the angle of incidence. Our method is able to distinguish vegetation from drivable surfaces such as streets. To predict the terrain type, we use a support vector machine (SVM). We avoid the need to label training data manually by labeling sets of example measurements in a self-supervised fashion by means of a pre-trained vibration-based terrain classifier. In addition to the system based on support vector machines, we presented a classification approach based on linear discriminant analysis (LDA). This system is around 400 times faster than the SVM-based approach and is especially designed for robots with limited computational resources. Both approaches have been implemented and evaluated in various real-world experiments. Our experiments show that the SVM-based approach is able to accurately detect low, grass-like vegetation with an accuracy of close to $100 \%$. The classification method based on LDA achieves accurate predictions, but in our experiments they were up to $4 \%$ worse than results of the SVM-based approach. In further experiments, we demonstrated that autonomous robots are able to navigate efficiently and safely in structured outdoor environments using our terrain classification method.

\section{Future Work}

In our experiments, training and testing data were collected on clear days by measuring try surfaces. Rain water or snow might affect the reflectivity - to what extent water affects classification results and how this can be detected is subject to future work.

The experiments presented in this paper were performed in early spring and in summer. Our approach was able to detect grass that was yellowed from being covered by snow in the preceding days. Future work could provide a more thorough evaluation of the impact of reduced chlorophyll on laser reflectivity.

The vibration-based classifier provided accurate training data in our experiments. If the training signal contained considerable noise, it might be possible to use the probabilistic output of the SVM-classifier to avoid uncertain training data.

\section{Acknowledgment}

This work has partly been supported by the German Research Foundation (DFG) under contract number SFB/TR-8 (A3) and by the European Commission under contract number FP7-231888-EUROPA and FP7248873-RADHAR.

\section{References}

[1] R. Myneni, F. Hall, P. Sellers, A. Marshak, The interpretation of spectral vegetation indexes, IEEE Transactions on Geoscience and Remote Sensing 33 (2) (1995) 481-486. 
[2] H. Moravec, Sensor fusion in certainty grids for mobile robots, AI Magazine (1988) 61-74.

[3] D. Wolf, G. Sukhatme, D. Fox, W. Burgard, Autonomous terrain mapping and classification using hidden markov models, in: Proc. of the IEEE Int. Conf. on Robotics \& Automation (ICRA), 2005.

[4] M. Hebert, N. Vandapel, Terrain classification techniques from ladar data for autonomous navigation, in: Proc. of the Collaborative Technology Alliances conference, College Park, MD., 2003.

[5] J.-F. Lalonde, N. Vandapel, D. Huber, M. Hebert, Natural terrain classification using three-dimensional ladar data for ground robot mobility, Journal of Field Robotics 23 (10) (2006) 839 861.

[6] J. Macedo, R. Manduchi, L. Matthies, Ladar-based discrimination of grass from obstacles for autonomous navigation, in: ISER 2000: Experimental Robotics VII, London, UK, 2001.

[7] R. Manduchi, Bayesian fusion of color and texture segmentations, in: Proc. of the Int. Conf. on Computer Vision (ICCV), 1999, p. 956.

[8] D. Bradley, R. Unnikrishnan, J. Bagnell, Vegetation detection for driving in complex environments, in: Proc. of the IEEE Int. Conf. on Robotics \& Automation (ICRA), 2007.

[9] B. Zhao, L. Tian, T. Ahamed, Real-time ndvi measurement using a low-cost panchromatic sensor for a mobile robot platform, Environment Control in Biology 48 (2) (2010) 73-79.

[10] B. Douillard, D. Fox, F. Ramos, Laser and vision based outdoor object mapping, in: Proceedings of Robotics: Science and Systems IV, Zurich, Switzerland, 2008.

[11] R. Manduchi, A. Castano, A. Talukder, L.Matthies, Obstacle detection and terrain classification for autonomous off-road navigation, Autonomous Robots, 18 (2003) 81-102.

[12] C. Wellington, A. Courville, A. Stentz, A generative model of terrain for autonomous navigation in vegetation, International Journal of Robotics Research 25 (12) (2006) 1287 - 1304.

[13] C. Brooks, K. Iagnemma, S. Dubowsky, Vibration-based terrain analysis for mobile robots, in: Proc. of the IEEE Int. Conf. on Robotics \& Automation (ICRA), 2005, pp. 3415-3420.

[14] E. DuPont, R. Roberts, C. Moore, M. Selekwa, E. Collins, Online terrain classification for mobile robots, in: Proc. of the Int. Mechanical Engineering Congress and Exposition Conference (IMECE), Orlando, USA, 2005.

[15] D. Sadhukhan, C. Moore, E. Collins, Terrain estimation using internal sensors, in: Proc. of the IASTED Int. Conf. on Robotics and Applications, Honolulu, Hawaii, USA, 2004.

[16] C. Weiss, H. Frohlich, A. Zell, Vibration-based terrain classification using support vector machines, in: Proc. of the IEEE/RSJ Int. Conf. on Intelligent Robots and Systems (IROS), 2006.

[17] C. Weiss, N. Fechner, M. Stark, A. Zell, Comparison of different approaches to vibration-based terrain classification, in: Proc. of the European Conf. on Mobile Robots (ECMR), Freiburg, Germany, 2007.

[18] M. Happold, M. Ollis, N. Johnson, Enhancing supervised terrain classification with predictive unsupervised learning, in: Proceedings of Robotics: Science and Systems, 2006.

[19] G. Grudic, J. Mulligan, M. Otte, A. Bates, Online learning of multiple perceptual models for navigation in unknown terrain, in: Field and Service Robotics, Springer, 2008, pp. 411-420.

[20] L. Matthies, M. Turmon, A. Howard, A. Angelova, B. Tang, E. Mjolsness, Learning for autonomous navigation: Extrapolating from underfoot to the far field, Journal of Machine Learning Research 1 (2005) 1-48.

[21] A. Angelova, L. Matthies, D. Helmick, P. Perona, Dimensionality reduction using automatic supervision for vision-based terrain learning, in: Proceedings of Robotics: Science and Sys- tems, 2007

[22] R. Hadsell, P. Sermanet, J. Ben, A. Erkan, M. Scoffier, K. Kavukcuoglu, U. Muller, Y. LeCun, Learning long-range vision for autonomous off-road driving, Journal of Field Robotics 26 (2) (2009) 120-144.

[23] H. Dahlkamp, A. Kaehler, D. Stavens, S. Thrun, G. Bradski, Self-supervised monocular road detection in desert terrain, in: Proc. of Robotics: Science and Systems (RSS), Philadelphia, USA, 2006.

[24] K. Wurm, R. Kümmerle, C. Stachniss, W. Burgard, Improving robot navigation in structured outdoor environments by identifying vegetation from laser data, in: Proc. of the IEEE/RSJ Int. Conf. on Intelligent Robots and Systems (IROS), 2009.

[25] B. Schölkopf, A. Smola, Learning with Kernels, MIT Press, 2002.

[26] C.-C. Chang, C.-J. Lin, LIBSVM: a library for support vector machines, http://www.csie.ntu.edu.tw/čjlin/libsvm (2001).

[27] J. Platt, Probabilistic outputs for support vector machines and comparisons to regularized likelihood methods, in: Advances in Large Margin Classifiers, 1999, pp. 61-74.

[28] V. Vapnik, Statistical Learning Theory, Wiley-Interscience, 1998.

[29] R. Baribeau, M. Rioux, G. Godin, Color reflectance modeling using a polychromatic laser range sensor, Pattern Analysis and Machine Intelligence, IEEE Transactions on 14 (2) (1992) 263 269.

[30] R. Triebel, P. Pfaff, W. Burgard, Multi-level surface maps for outdoor terrain mapping and loop closing, in: Proc. of the IEEE/RSJ Int. Conf. on Intelligent Robots and Systems (IROS), 2006.

[31] E. Alpaydin, Introduction To Machine Learning, MIT Press, 2004.

[32] G. Grisetti, C. Stachniss, W. Burgard, Improved techniques for grid mapping with rao-blackwellized particle filters, IEEE Transactions on Robotics 23 (1) (2007) 34-46.

[33] C. Stachniss, G. Grisetti, GMapping project at OpenSLAM.org, http://openslam.org/gmapping.html (2007). 